\title{
3-31-2018
}

\section{A Sketch-based Rapid Modeling Method for Crime Scene Presentation}

Pu Ren

Beijing Normal University, rp_apei@163.com

Wuyang Shui

Beijing Normal University, sissun@126.com

Jin Liu

Institute of Forensic Science, Ministry of Public Security

Yachun Fan

Beijing Normal University

Wenshuo Zhao

General Office, Ministry of Public Security

Follow this and additional works at: https://commons.erau.edu/jdfsl

See next page for additional authors

Part of the Other Computer Engineering Commons

\section{Recommended Citation}

Ren, Pu; Shui, Wuyang; Liu, Jin; Fan, Yachun; Zhao, Wenshuo; and Zhou, Mingquan (2018) "A Sketch-based Rapid Modeling Method for Crime Scene Presentation," Journal of Digital Forensics, Security and Law.

Vol. 13 : No. 1 , Article 8.

DOI: https://doi.org/10.15394/jdfsl.2018.1484

Available at: https://commons.erau.edu/jdfsl/vol13/iss1/8

This Article is brought to you for free and open access by

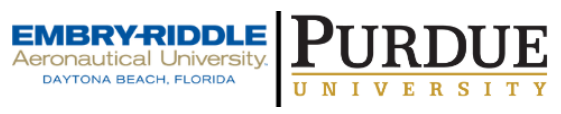
the Journals at Scholarly Commons. It has been accepted for inclusion in Journal of Digital Forensics, Security and Law by an authorized administrator of (c)ADFSL Scholarly Commons. For more information, please contact commons@erau.edu. 


\section{A Sketch-based Rapid Modeling Method for Crime Scene Presentation}

\section{Cover Page Footnote}

footnote: This article is the extended version of the conference paper which was published on the 9th EAI International Conference on Digital Forensics \& Cyber Crime (ICDF2C) on Oct.9-11, 2017.

acknowledgement: The authors would like to thank the anonymous reviewers. Special thanks to all the members of SweetHome3D project. This work is supported by the open subject of the key laboratory of traces of science and technology of Ministry of Public Security (No. 2017FMKFKT03), the National Natural Science Foundation of China (No. 61402042, 61672103) and the National Key Technology Research and Development Program of China (No. 2017YFB1002804).

\section{Authors}

Pu Ren, Wuyang Shui, Jin Liu, Yachun Fan, Wenshuo Zhao, and Mingquan Zhou 


\title{
A SKETCH-BASED 3D MODELING METHOD FOR 3D CRIME SCENE PRESENTATION
}

\author{
Pu Ren ${ }^{1,2}$; Wuyang Shui ${ }^{1,2}$; Jin Liü; Yachun Fan ${ }^{1,2}$; Wenshuo Zhao ${ }^{4}$; \\ and Mingquan $\mathrm{Zhou}^{1,2}$
}

1. College of Information Science and Technology, Beijing Normal University, Beijing, China 2. Engineering Research Center for Virtual Reality Applications, MOE, Beijing, China 3. Institute of Forensic Science, Ministry of Public Security, Beijing, China

4. General Office, Ministry of Public Security, Beijing, China

\begin{abstract}
The reconstruction of a crime scene plays an important role in digital forensic application. This article integrates computer graphics, sketch-based retrieval, and virtual reality (VR) techniques to develop a low-cost and rapid 3D crime scene presentation approach, which can be used by investigators to analyze and simulate the criminal process. First, we constructed a collection of 3D models for indoor crime scenes using various popular techniques, including laser scanning, imagebased modeling and geometric modeling. Second, to quickly obtain an object of interest from the 3D model database, a sketch-based retrieval method was proposed. Finally, a rapid modeling system that integrates our database and retrieval algorithm was developed to quickly build a digital crime scene. For practical use, an interactive real-time virtual roaming application was developed in Unity 3D and a low-cost VR head-mounted display (HMD). Practical cases have been implemented to demonstrate the feasibility and availability of our method.
\end{abstract}

Keywords: indoor crime scene presentation, 3D model database, sketch-based retrieval, rapid modeling, immersive display

Note: This article is the extended version of the conference paper which was published on the 9th EAI International Conference on Digital Forensics \& Cyber Crime (ICDF2C) on Oct.9-11, 2017

\section{INTRODUCTION}

In case of criminal incidents, the first phase of inspection and investigation is to rapidly record a complete, objective crime scene representation without erroneous information. In comparison to traditional methods, i.e., verbal descriptions, hand-drawn sketches, photos, videos, etc., the use of $3 \mathrm{D}$ crime scene presentation is more intuitive and effective.

Although 2D photos, videos, and spherical photographs have been used in the courtroom, there are challenges in using these resources to describe and present the crime scene because these resources only provide information from a given view (Ebert, 2014; Tung, 2015). 3D presentation enables investigators to virtually experience the crime scene, measure the distances between different objects and simulate the criminal activity. In a more extreme case, the real crime scenes cannot be entered again, such as the criminal cases happened many years ago. Therefore, the virtual representation of the crime scenes became very essential in practice. In this paper, 
a rapidly, effective and easy-to-use method of presenting a 3D crime scene was proposed.

We have observed that in traditional practical crime investigations, policemen are often tasked with recording the indoor layout in hand-drawn sketches. Inspired by this observation and existing techniques, this article presents an effective method to build a digital indoor crime scene to generate the spatial relationships of objects for crime analysis and presentation. Additionally, rather than relying on sketches of the layout and objects, investigators can directly obtain a 3D scene using our system and explore this virtual crime scene anywhere and at any time.

\section{LITERATURE REVIEW}

In recent studies of forensic investigation, the use of a terrestrial laser scanner (TLS) or RGB-D camera has become a popular technique for the acquisition of 3D models of indoor crime scenes and evidence (Sansoni, 2011). The time-of-flight laser scanner, structure-light scanner and triangulation-based scanner have been used to acquire both the large-scale geometric information of crime scenes and the small-scale geometric information of evidence and victims (Ma, 2010; Holowko, 2016; González-Jorge, 2015; Buck, 2013). These noninvasive scanners can be used to accurately generate a digital 3D scene model after registration of range images and geometric mesh processing.

However, these devices are always expensive and the generation of digital models from unorganized point clouds is always based on manual and expert procedures. It is difficult to widely promote the application of this technique in local police stations. To address this problem, another type of modeling method is to produce a realistic $3 \mathrm{D}$ digital model from a series of images, the procedures for which comprise camera calibration, dense point-cloud computation, surface reconstruction and post- processing (Gibson, 2000; Bostanci, 2015; Leipner, 2016). The quality of a reconstructed model depends largely upon the algorithm used to calculate feature correspondence and camera calibration.

Nevertheless, it is time consuming and expensive to use these optical device-based methods. More importantly, the 3D reconstruction techniques could only be used for the rebuilding of existing crime scenes. We adopted these techniques to record some real 3D data of important objects in our research.

Considering investigators would like to quickly represent the spatial information of crime scenes, the use of professional geometric 3D modeling software, such as AutoCAD, 3Ds Max, SketchUp, etc., is the most common, intensive and powerful technique in the practice application of $3 \mathrm{D}$ crime scene representation (Clair, 2012; Howard, 2000; Maksymowicz, 2014). However, one potential problem of this technique is that investigators have no professional training or experience in producing 3D models using software. It increases the labor costs in practice to engage extra artists to complete the 3D scene modeling work. In addition, the modeling task is tedious and time consuming, because hundreds of $3 \mathrm{D}$ models should be generated for a crime scene.

In sum, existing methods cannot simultaneously meet the requirement of rapidly, accurately and efficiently generating crime scenes at a low cost. This paper realized a system for rapidly rebuilding crime scenes based on hand-drawn sketches to facilitate onsite investigations for the police. Investigators are able to rapidly rebuild a $3 \mathrm{D}$ crime scene through a simple interface and control the model size by adjusting parameters. As for decisive evidence, a handheld 3D scanner, or just a set of photos, can be used to acquire the precise surface data of the objects. The 3D scene data can be rendered in a virtual 
environment in real time, and it can also be import into other engine to realize VR roaming effect.

\section{DATASET}

The high quality and large number of 3D models in the dataset improved the presentation of the $3 \mathrm{D}$ crime scene. By collecting more categories and a larger number of models in each category, the user can retrieve much more appropriate objects. In this study, we utilized different techniques to construct a specialized 3D model database including crime-related models and common models in indoor scenes.

Although most of the 3D data are collected from the public online source, we have adopted different techniques to generate the real 3D data. First, the most accurate data could be generated by 3D laser-scanning method. Second, in order to reduce the cost, an imagebased 3D reconstruction algorithm has been used.

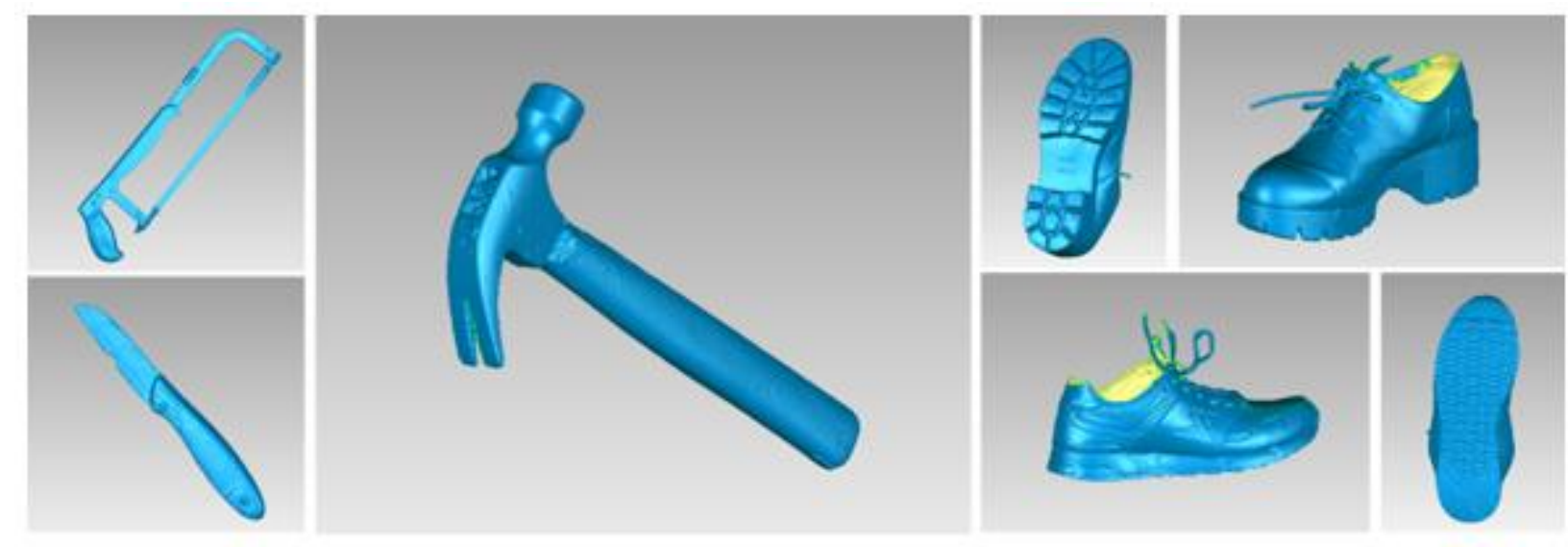

Figure 1. 3D models capture by laser scanner. (Crime-fighting tools and shoe soles.)

\section{$3.13 \mathrm{D}$ reconstruction techniques}

Some objects that appear in a crime scene are unique and cannot be replaced by the existing models in the database. Thus, we used laser-scanning and image-based modeling techniques to capture real data of critical evidence. We used a high-precision hand-held laser scanner HandySCAN 700 (Creaform Company, Canada) to reconstruct 3D models of objects. The benefit of this laser scanner is that it automatically produces complete single mesh models, without the procedure of manual range-image registration in other scanners (e.g., Vivid 910 laser scanner). Fig. 1 shows some examples of 3D models captured by laser scanner. In addition to some crime tools there are two shoes and soles (usually very important evidence) in this figure. The main benefit of this technique is that it acquires high-quality 3D models with more details. However, the high cost of the device prohibits its widespread use.

The structure-from-motion technique is used as a substitute in cases in which the laser scanner cannot be used. This technique utilized the principle of two eyes parallax in computer version research, generating $3 \mathrm{D}$ space data from multiple $2 \mathrm{D}$ images. Inputting a set of photos of the object, a powerful and free tool called VisualSFM $(\mathrm{Wu}$, 2013; Jancosek, 2011) was used to generate a dense 3D point cloud with color. Fig. 2 shows 
some examples of 3D models acquired by the SFM technique.

To construct a mesh model from point clouds, the open-source software Meshlab was used to realize surface reconstruction and postprocessing (Cignoni, 2008). In Meshlab, the normal vector at every point was estimated and the Poisson surface reconstruction algorithm was used to generate a mesh model. This technique is easier to use than $3 \mathrm{D}$ scanning since it is convenient to acquire multiple photos for evidence.

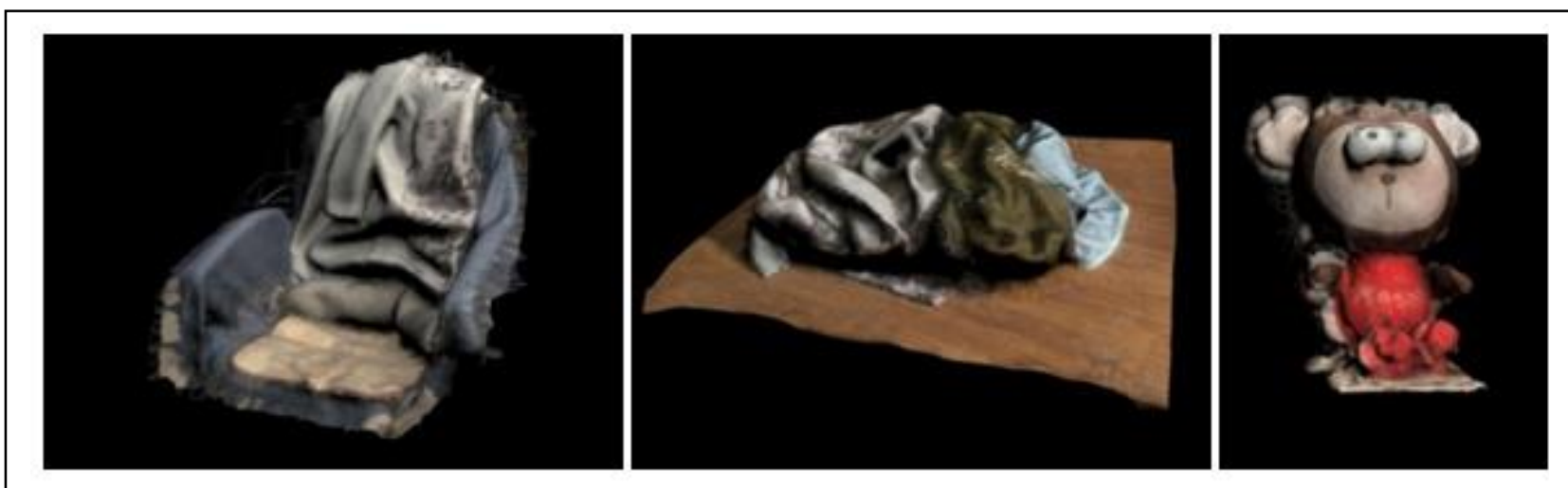

Figure 2. 3D models reconstructed by SFM technique. Left: a chair with clothes on it. Middle: a mass of clothes. Right: a plush toy.

\section{$3.23 D$ model dataset}

To extend the number and categories of 3D models, we collected 3D models from online public-domain galleries, such as the Google 3D Warehouse, which offers thousands of models online (Clair, 2012), and the public database from literature (Fisher, 2012). As a supplement, some models were created by our professional designers using geometric modeling software. Figure 3 shows some examples of 3D models created using commercial software. Figure 3-a shows 3D models of furniture. Moreover, the victim's posture plays a significant role in crime investigation. Thus, multiple types of human models with various postures are available in our database to ensure that the most similar one to the real situation would be found (Figure 3-b). In summary, we offered a total of 2,564 models and 18 categories in our collection, including windows, doors, home appliances, furniture, human bodies, and criminal tools. Table 1 lists the main types of models generated from different sources in our database. The decisive evidence refers to some material evidence which play a decisive role in the real investigation. 


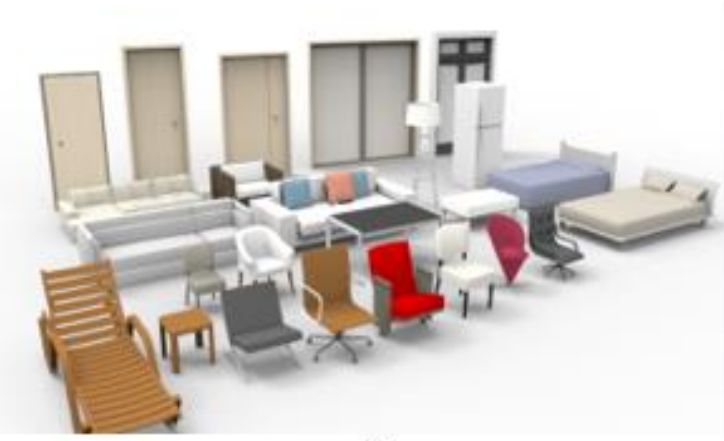

(a)

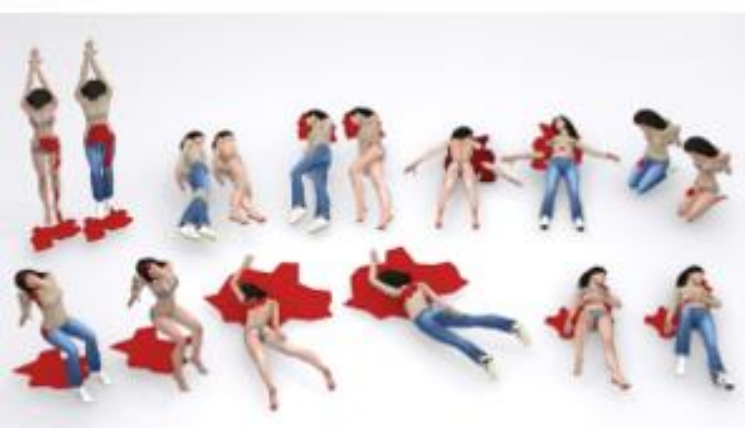

(b)

Figure 3. A collection of 3D models in the crime scene. (a) Furniture models with various shapes. (b) Human models with various postures.

Table 1.

$3 D$ model database.

\begin{tabular}{|ccccccc|}
\hline Source & Online libraries & $\begin{array}{c}\text { Open } \\
\text { literature }\end{array}$ & $\begin{array}{c}\text { Software } \\
\text { modeling }\end{array}$ & Laser scanning & $\begin{array}{c}\text { Image-based } \\
\text { modeling }\end{array}$ \\
\hline Indoor models & 1,123 & 659 & 361 & 0 & 18 & 6 \\
\hline Murder related & 240 & 0 & 135 & 12 & 5 & 393 \\
\hline Decisive evidence & 0 & 0 & 2 & 3 & 5 \\
\hline Total & & & & & 2,564 \\
\hline
\end{tabular}

\section{METHOD}

Sufficient model data makes it possible for users to reuse models, and a good retrieval algorithm is also needed to ensure the quickness and accuracy of model selection. Users can traverse the model database looking for the target model, which is time consuming. The traditional retrieval way is the text-based method; however, it is relying on the label of $3 \mathrm{D}$ models. And in most time, it is just very easy to sketch the object you want, rather than describe it by some single words. Thus, an intuitive sketch-based method is much more suitable for the current application.

\subsection{Sketch-based indoor scene modeling}

It is a challenge to rapidly obtain the appropriate model from large collections of $3 \mathrm{D}$ models since public collections are often insufficiently annotated (Eitz, 2012). To address this problem, the content-based retrieval technique is a powerful and effective tool. The general idea of the sketch-based method is that the user retrieves the desired model through the input of a hand drawing.

In this study, our sketch-based retrieval was divided into two stages: offline index and online query. To save time in a real-time query, we perform the computation offline as much as possible. Figure 4 shows the 
framework of our algorithm and Figure 5 shows one of the retrieval results. The input sketch is shown on the upper left, and the first couch model is the most similar model in the database. The key steps of our algorithm are described below.

Step 1: Line-drawing calculation. The essence of 3D model retrieval is to evaluate the similarity between hand-drawn sketches and models. Towards this end, 3D information should be first projected into $2 \mathrm{D}$ images. $2 \mathrm{D}$ line drawings are extracted from 102 viewpoints of geodesic geometry for each model in the database, reflecting the visual information as much as possible. Different from most of the previous line-drawing extraction algorithms, which were only available for homogeneously distributed dense meshes, we proposed a depth map-based difference-ofGaussian (DoG) method to extract lines, including the boundaries and creases formed by differences in depth. The information in the depth buffer is used to generate depth images with different Gaussian parameters, called D1 and D2, and the line drawing is generated by calculating the inverse of binary image, i.e., the Gaussian difference of D1 and D2. To overcome the saw tooth noise problem, the Bezier curve approximation is used to perform the line stylization and obtain a smooth contour line (Qian, 2017).

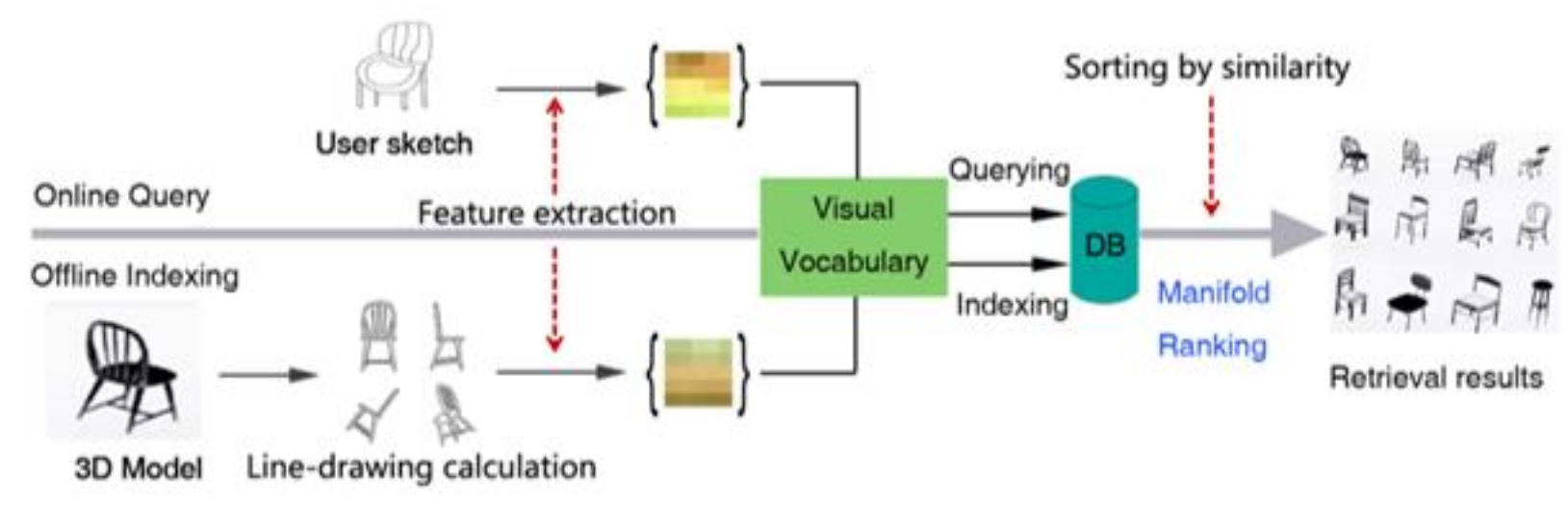

Figure 1. The framework of our sketch-based retrieval algorithm.
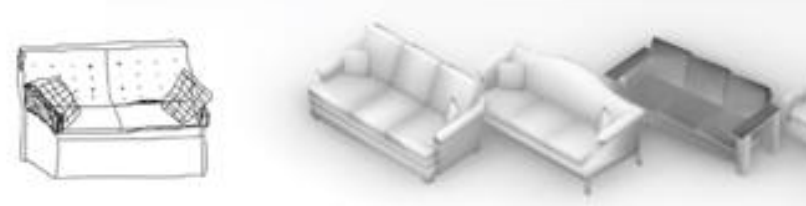

Figure 2. Retrieval Results using sketch-based technique. The left figure shows an input of hand drawing by user. Right figure shows the first nine most similar models.

Step 2: Feature extraction. We used a spatial, high-dimensional and local featuredescription algorithm to extract features. As a type of short-time Fourier transform, Gabor transform is sensitive to the edges of an image. It can provide good selection for frequency and 
orientation. In addition, the Gabor filter is similar to the mammalian visual system in the expression of frequency and orientation. It is suitable for sketch retrieval requests. Thus, we use an algorithm called the Space Pyramid of Gabor Local Feature Extraction and obtain better results (Eitz, 2012). Next, we define a set of Gabor filters $g_{i}(i=1,2, \ldots, k)$ to calculate the basic transform of the feature region, and the information on the spatial distribution is obtained in the space pyramid of the image. For each $g_{i}$ and image $I$, a convolution computation is employed to obtain a response image $R_{i}$ :

$$
R_{i}=\left\|\operatorname{idft}\left(g_{i} * \operatorname{dft}(I)\right)\right\|
$$

where $*$ denotes point-wise multiplication, and the function $\operatorname{dft}()$ and $\operatorname{idft}()$ respectively denote the discrete Fourier transform and the inverse discrete Fourier transform. A gridsampling method is used for feature-point sampling. For a local shape feature, we divide the region into $n \times n$ cells $C_{\pi}$, in which $r$ and $c$ respectively denote the row and column coordinates. The descriptor in the $i$ direction is a feature vector of size $n \times n$.

$$
F(r, c, i)=\frac{1}{N} \sum_{(x, y) \in C_{r c}} R_{i}(x, y)(2)
$$

where $N$ denotes the sample number for which $R_{i}(x, y) \neq 0$ in the $i$ direction. The local feature is a $k \times n \times n$ dimensional vector.

Step 3: Sorting by similarity. We employed TF-IDF (Zobel, 2006) to calculate the similarities of feature vectors among various models. In an ideal situation, the first- place model is the desired model, and then we import it into the crime scene. As the retrieval algorithm is not the focus of this work, readers can find more complete algorithm details in our other work (Qian, 2017).

\subsection{System interface}

Our system was developed on the basis of open-source software SweetHome3D licensed under the GNU General Public License (eTeks, 2017). We developed the sketch-based algorithm in Microsoft Visual Studio in $\mathrm{C}++$ and exported to a Dynamic-link library (dll) file. This dll file was imported as a plugin on a new user interface implementing sketch-based function. Our proposed 3D crime scene representation system contains five modules (see Figure 6): (a) the user draw a sketch of the desired from any viewpoint at the left-top area, and the retrieval results are displayed on the bottom, sorted according to similarity; (b) a $2 \mathrm{D}$ plan view that referring to the ichnography, can be loaded into this module as a base map where the layout of the indoor scene and the selected model can be transformed to the designated location (translation, rotation and scale); (c) a 3D display view where the $3 \mathrm{D}$ model scene corresponding to the $2 \mathrm{D}$ plan view is displayed and the user can observe the scene from any view and roam the scene as a virtual character; (d) a model classification and viewing tree in which a collection of $3 \mathrm{D}$ models is classified according to the given classification scheme and every model can be selected and viewed; and (e) a scene management interface where the parameters and statues of every model can be edited. 


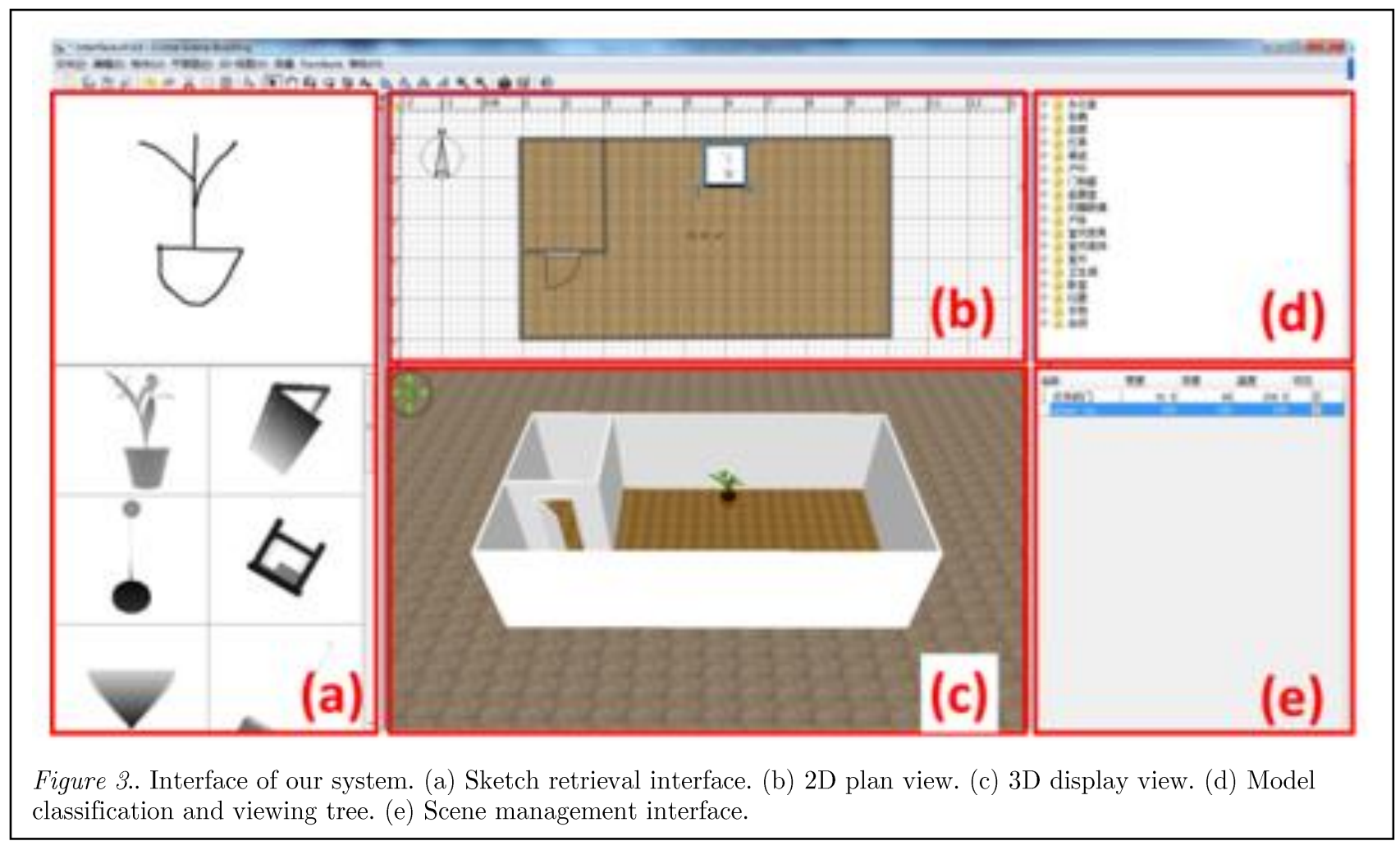

The major advantage of our system is that simple 2D sketches can be rapidly transformed into a complex 3D crime scene. In the first stage, users need to draw a sketch on the sketch-retrieval interface to obtain the desired objects from the database directly. Second, the retrieved models are placed on the $2 \mathrm{D}$ plane view and the corresponding $3 \mathrm{D}$ scene is simultaneously rendered on the 3D display view. The user can either view the $3 \mathrm{D}$ scene from any directions or roam in it anywhere and at any time. In the scene-management module, users can adjust the parameters and status of the chosen model, including visibility, texture, object position and size. Another notable feature of our system is that it is easy to measure the distance between two objects (Figure 7 ). In the 3D scene shown in (a), the distance between the body and the murder weapon can be easily measured by connecting them with a line as shown in (b). Finally, the $3 \mathrm{D}$ crime-scene representation can be exported as a standard .obj files. 


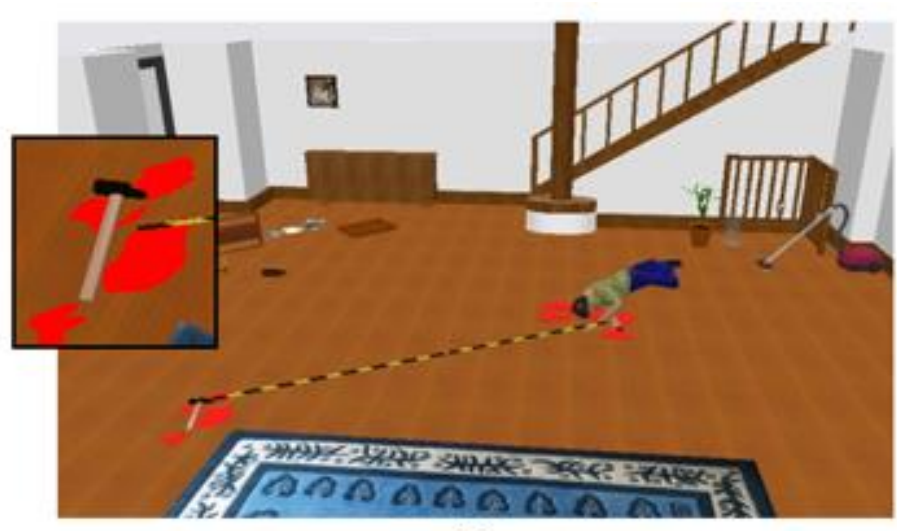

(a)

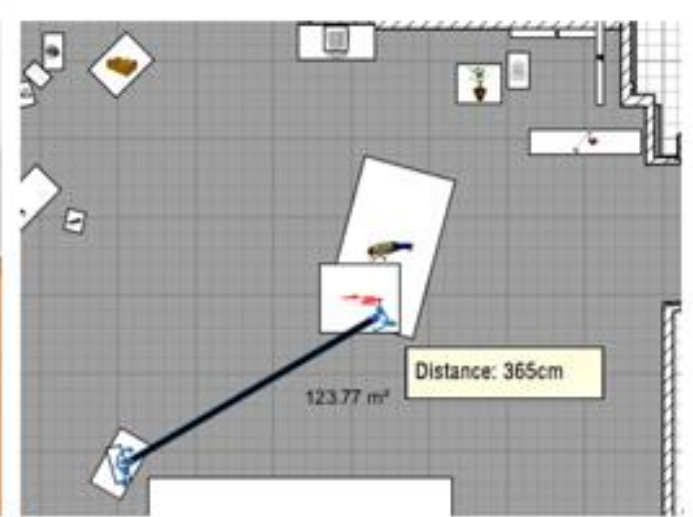

(b)

Figure 4. The distance measuring function of the system.

\subsection{Immersive display}

As a supplement to the virtual roaming function provided by our system, a helmetmounted display (HMD) device, such as HTC Vive (HTC and Valve), can be used to increase the immersive VR effect. This device has a high resolution of $2160 \times 1200$ pixels, resulting in a resolution of $1200 \times 1080$ pixels for each eye. HTC Vive provides a pair of handheld controllers and positioning-system tracking display, which allow the user to move within a certain scope and interact with the virtual scene using the handle (Ebert, 2014). These advantages are very suitable for the action simulation and virtual representation of crime scenes.

Utilizing the encapsulated SDK in the cross-platform game engine Unity3D (Unity Technologies, San Francisco, USA), we developed a VR roaming system for crime scenes. The 3D model scene built with our proposed sketch-based indoor-scene system can be imported into Unity3D and real-time interactive virtual-experience systems are developed using Microsoft Visual Studio C\#. In comparison to the traditional PC screen, the immersive display offers a larger viewing volume and more realistic experience. With the headset and handle, the investigator can enter the crime scene in digital space, observe 3D models of physical evidence, and analyze the case from a more intuitive angle.

\subsection{Ilustrative example}

To demonstrate the availability and effectiveness of our system, we quickly present an example of how to create an acceptable 3D crime scene for presentation from series of $2 \mathrm{D}$ sketches. We took a criminal case that happened fifteen years ago as an example; specifically, three adults and one child were killed in an apartment (Figure 8). The only remaining visual documents for this case are several low-resolution photos and a handdrawn floor plan of the crime scene.

Using our system, we have rapidly reconstructed this indoor scene and develop a roaming system in VR space. The result of our research has assisted the investigators to simulate the criminal's actions in the crime, analyze the motivation and walking route, and eventually solve this case. The analysis results have been confirmed by the criminals after the case was solved. 


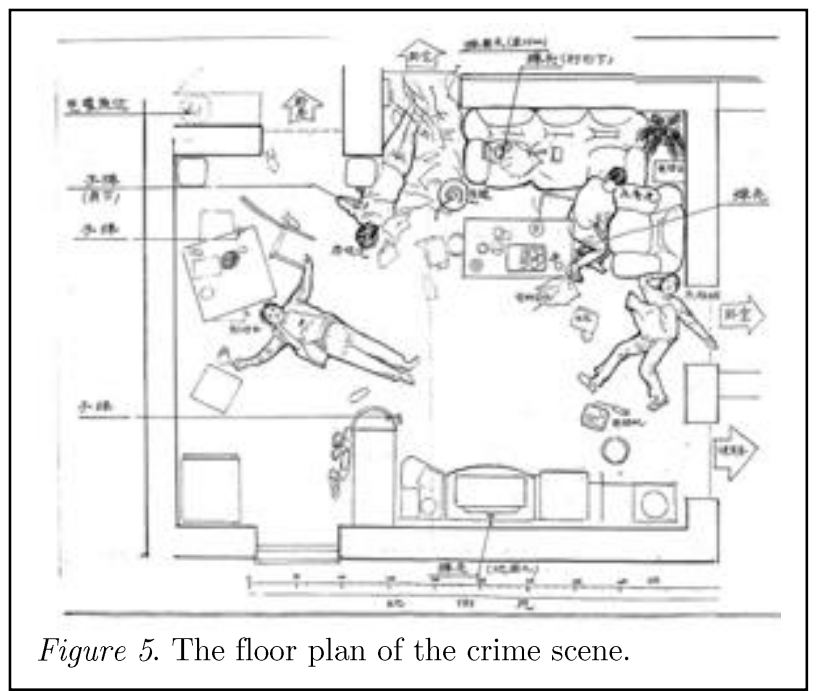

In the very few visual records of this case, the floor plan is a crucial piece of physical evidence for crime-scene reconstruction. By importing the hand-drawn floor plan into our system as a base map, we obtained the measurements and layout information of $3 \mathrm{D}$ models. Then, the user dragged the mouse to draw 2D shapes of walls on the 2D plan view according to the tracing lines of the imported base map, and the 3D scene was rendered in the 3D display view in real time (Figure 9-a). To enable the accurate representation of the $3 \mathrm{D}$ crime scene, the user scaled each shape according to the real measurement values, i.e., user defined the length, height, and width of the indoor scene.
The main contribution of our approach is the sketch retrieval function in this system. Because of this part of work, the investigators can rapidly find the objects which are in the similar shape contour with the objects in the base map without any semantic information of the 3D models.

Figure 9-b shows an example of retrieving the sofa. The retrieved model was imported into the $2 \mathrm{D}$ plan view and placed at the designated location, according to the base map. The 3D view was rendered simultaneously. The $3 \mathrm{D}$ scene can be viewed from any view and roamed at any time during the modeling procedure. All the colors and textures of imported models can be exchanged according to the real scenario. These steps were repeated until all objects were imported and located at the correct locations.

Figure 10 shows the 3D rendered effect of this crime that was constructed, which consisted of a total of 58 digital models and was rebuilt in a total of 13 minutes. The images are also rendered in our system, without any other plugins. The top two screenshots are bird's eye view and the bottom two are roaming perspective.

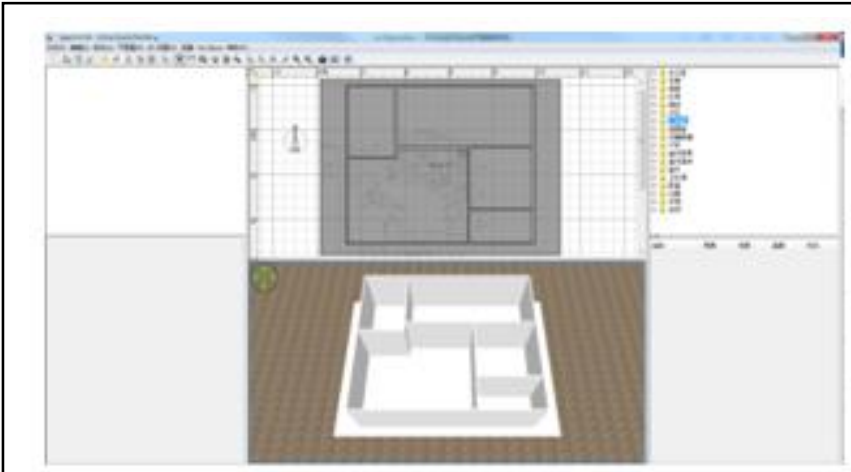

(a)

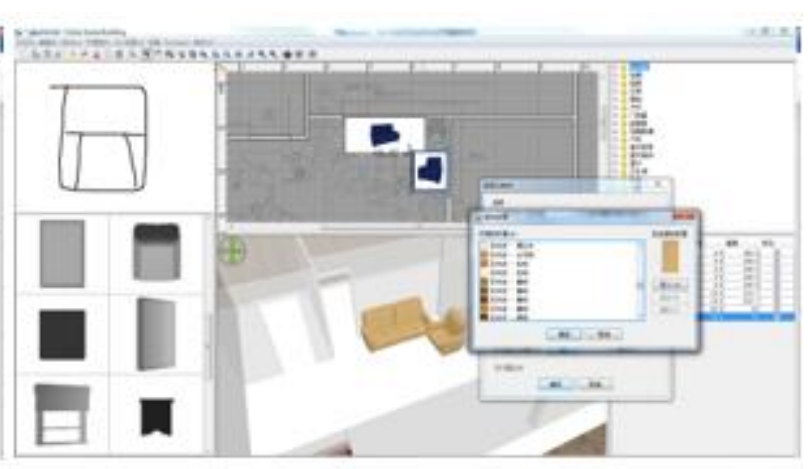

(b)

Figure 6. Sketch-based modeling for the indoor crime scene. (a) Reconstruct the walls based on imported base map. (b) Retrieving 3D models by sketches and changing the textures. 

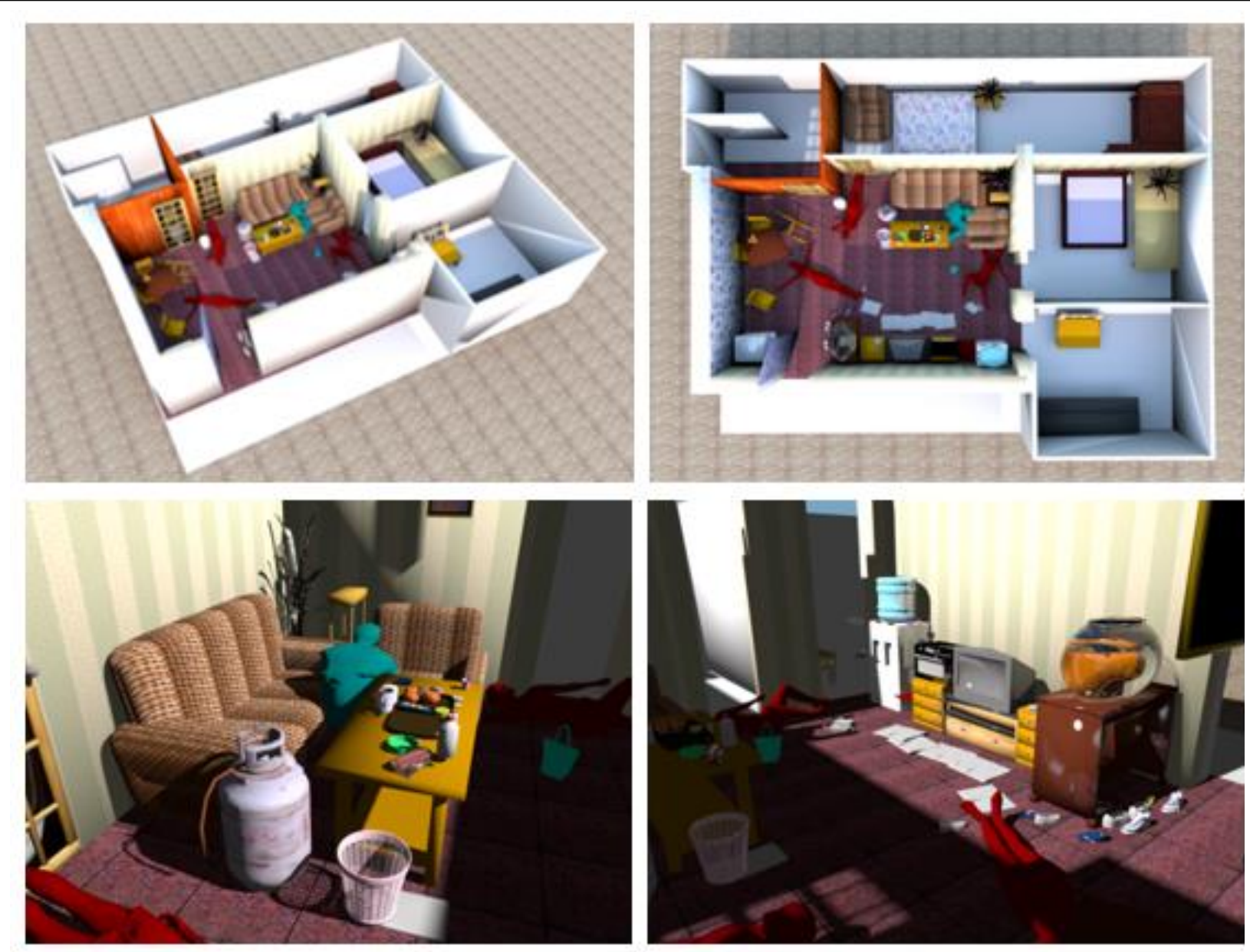

Figure 7. The creation of 3D indoor crime scene using our system.

To virtually experience this crime scene in an immersive display, the created crime scene can be exported as a standard .obj file, and an interactive virtual roaming system was developed in Unity3D, enabling investigators to experience the crime scene from a fixed viewpoint or different views to examine more details of the evidence of interest. The handles of HTC Vive are utilized to simulate a murder tool in the application. Figure 11 shows the immersive roaming effect using HTC Vive. In this application, the user can intuitively experience and analysis the shooting and walking routes of the criminal in the virtual space. 

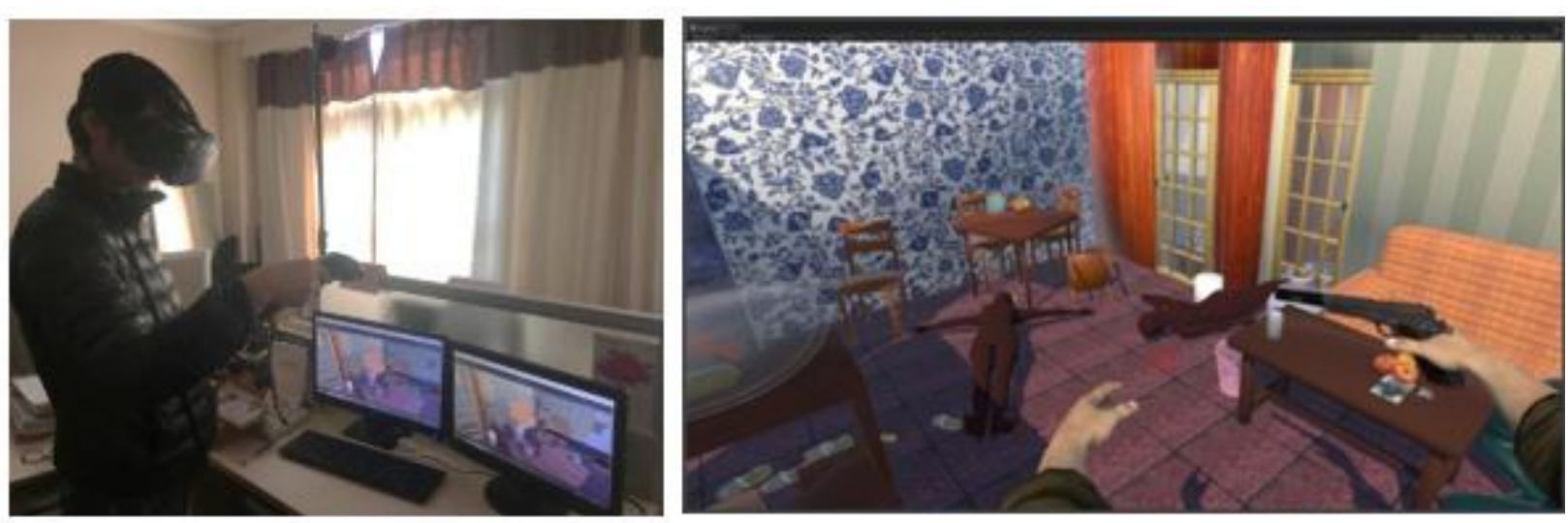

Figure 8. Interactive crime scene experience in the immersive display using HTC Vive. Left: Photo of the experiencer. Right: Real-time screenshot.

\section{EVALUATION}

Besides the practice application in the $3 \mathrm{D}$ virtual reconstruction of the non-existing real crime scenes, our method can also be used in the daily works for investigators. Compared with the traditional image or video recording ways, the $3 \mathrm{D}$ representation is obviously more intuitive. And compared with the directly $3 \mathrm{D}$ scene scanning method, our method is much more convenient and effective.

In order to demonstrate the effectiveness of our methods, we have done a comparison experiment between the 3D scanning method and our rapid modeling method. Figure 12 and Table 2 show the results of the 3D scene generated by scanning method and our approach. The point cloud in left is the real scan data which needs to be converted into mesh model for VR use. The right figure in Figure 12 shows our result. By using our system, a simplified 3D mesh model can be directly reconstructed without any other postprocessing. Our method ignored inessential details to generate the $3 \mathrm{D}$ scene rapidly.

In Table 2, the modeling time, number of the meshes in the final scene, and the frame rate when roaming in these two scenes are listed for comparison. We concluded that creating an acceptable model that is not completely accurate to record the spatial information of objects in a crime scene is an available and valuable approach for further investigation and presentation. 

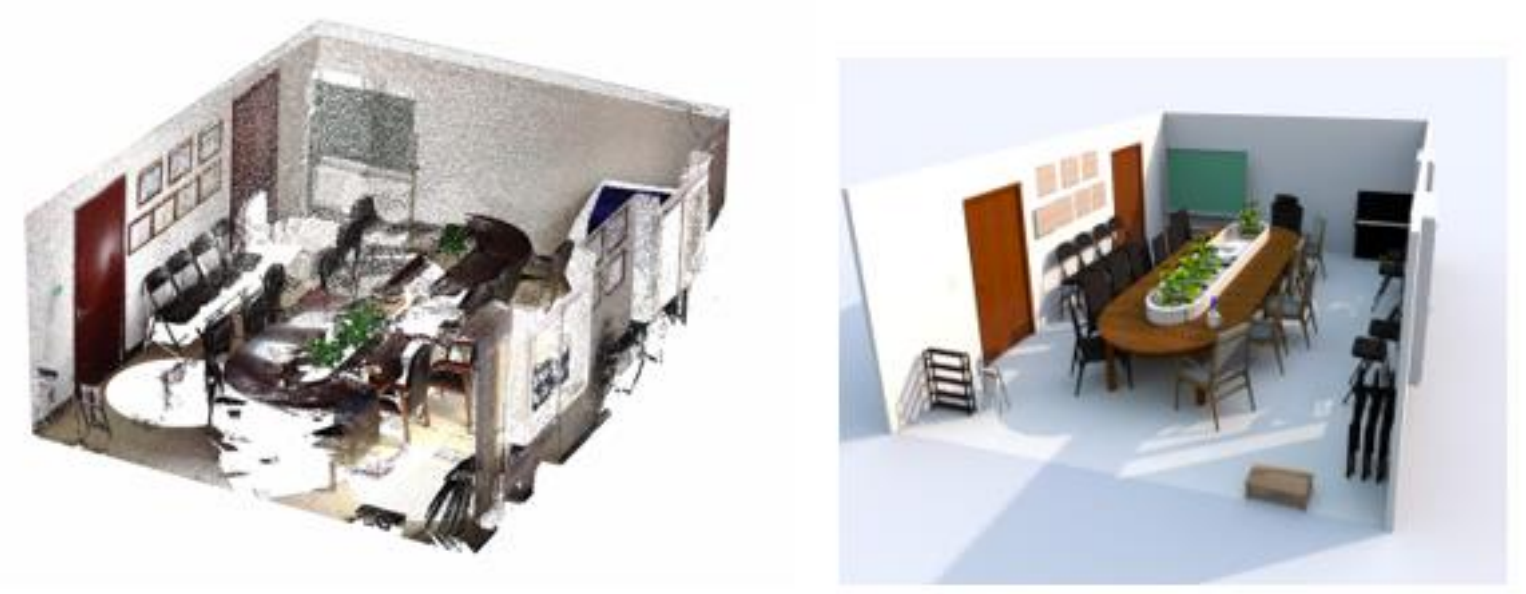

Figure 12. Comparison with the 3D scanning scene. Left: Point cloud data generated by 3D scanning. Right: Mesh model generated in our system.

Table 2.

Statistic data of the comparison experiment.

\begin{tabular}{|ccc|}
\hline & 3D Scanning & Our approach \\
\hline Modeling time (min) & 150 & 20 \\
\hline The number of the mesh & $2,000,000$ & 311,882 \\
\hline Frame rate in a VR scene (fps) & 9.5 & 67 \\
\hline
\end{tabular}

\section{DISCUSSION}

In comparison to traditional recording approaches, e.g., 2D hand-drawn sketches, photos, videos, 3D digital scenes and animations have been used to enhance clarity and understanding in crime-scene investigation (Ma, 2010; Holowko, 2016; Buck U, 2013; Maksmowicz, 2014). Although the laserscanning technique provides life-like and accurate 3D models of crime scenes, it has limitations for wide promotion. On one hand, the devices are expensive. On another hand, it is still a great challenge to convert dense triangle meshes (more than hundreds of thousands of triangle meshes) into a simplified model to meet the requirement of real-time virtual experience. Another limitation is that many crime scenes cannot be entered after the criminal incident. In this situation, investigators need to model the scene manually. Clair et al. introduced the application of the easy-to-use modeling software SketchUp to generate 3D indoor crime-scene models (Chair, 2012). It was an extremely time-consuming process to generate every model. To solve this problem, Howard et al. constructed a collection of 3D models and generated the non-critical areas of digital crime scenes using the existing models (Howard, 2000).

One of the contributions of our work is utilizing multiple techniques to construct a large collection of $3 \mathrm{D}$ models for indoor scene. The 3D models with complex structures and 
more details, e.g., guns, knives, shoe soles, etc., were generated by laser-scanning or imagebased modeling techniques. To enable real-time rendering of this model in Unity 3D, the opensource Meshlab software was used to convert this 3D model to a simplified model and texture.

Another contribution of our work is the sketch-based model retrieval. Because of the large number of models in the collection, investigators have become more comfortable and it has become easier to generate a crime scene by our method. However, it is also a challenge for investigators to rapidly obtain the desired model from datasets. Compared with the traditional text-based retrieval methods, the 2D hand-drawn sketch-based approach has much more potential in our application since few annotations are available for each 3D model. In this paper, we propose a sketch-based approach to obtain a suitable model with geometric structure similar to that of the real object from the database. To achieve real-time retrieval, we have extracted and archived the feature lines of every model in advance. Because of this main part of our work, crime scenes can be rebuilt quickly using police's hand-drawn sketches rather than those of professional designers.

To better provide a crime-scene model, a multi-participant, large-screen stereoscopic projector system (Howard, 2000), an immersive display VR headset (Ebert, 2014), and the augmented-reality technique have been used in previous studies (Gee, 2010). In our article, we provided a general system covering the functions of rebuilding an indoor crime scene and rendering it in $3 \mathrm{D}$ space. To realize immersive display, the rebuilt scene model was imported into Unity 3D to develop a VR roaming system in HTC Vive.

There are still some limitations of our approach. We have successfully extended the crime scene model database, but both the number of category in the database and the number of models in each category are insufficient for crime-scene presentation. We will continue to generate multiple kinds of models to build an extensive crime-scene model database. A system allowing the user to retrieve multiple $3 \mathrm{D}$ models at the same time is also helpful in practice.

\section{CONCLUSION}

Based on the 3D model collection, a sketchretrieval based modeling system was developed. Because of the TF-IDF algorithm, our method achieves high retrieval accuracy and time efficiency. Using our approach, police investigators can rapidly record the spatial relationships of objects while constructing a digital model of the indoor crime scene. In comparison to laser-scanning and softwaremodeling techniques, the main advantage of our method is that it is low cost and rapid, making it very suitable for criminal investigation. In terms of digital forensics and educational training applications, the immersive-display we developed has broad prospects because of the VR interactive experience in the crime scene.

\section{ACKNOWLEDGEMENT}

The authors would like to thank the anonymous reviewers. Special thanks to all the members of SweetHome3D project. This work is supported by the open subject of the key laboratory of traces of science and technology of Ministry of Public Security (No. 2017FMKFKT03), the National Natural Science Foundation of China (No. 61402042, 61672103) and the National Key Technology Research and Development Program of China (No. 2017YFB1002804). 


\section{REFERENCES}

Bevel T, Gardner RM. (2008). Bloodstain pattern analysis with an introduction to crime scene reconstruction: $C R C$ Press.

Bostanci E. (2015). 3D Reconstruction of Crime Scenes and Design Considerations for an Interactive Investigation Tool. Computer Science. 896-900.

Buck U, Naether S, Räss B, Jackowski C, Thali MJ. (2013). Accident or homicidevirtual crime scene reconstruction using $3 \mathrm{D}$ methods. Forensic science international. 225:75-84.

Clair ES, Maloney A, Schade A. (2012). An Introduction to Building 3D Crime Scene Models Using SketchUp. Journal on the Association for Crime Scene Reconstruction. 18:29-47.

Cignoni P, Callieri M, Corsini M, Dellepiane M, Ganovelli F, Ranzuglia G. (2008). MeshLab: An Open-Source Mesh Processing Tool. Eurographics Association. 129-136.

Ebert LC, Nguyen TT, Breitbeck R, Braun M, Thali MJ, Ross S. (2014). The forensic holodeck: an immersive display for forensic crime scene reconstructions. Forensic science, medicine, and pathology. 10:623-6.

Eitz M, Richter R, Boubekeur T, Hildebrand K, Alexa M. (2012). Sketch-based shape retrieval. ACM Trans on Graphics. 31: 110.

Fisher M, Ritchie D, Savva M, Funkhouser T, Hanrahan P. (2012). Example-based synthesis of $3 \mathrm{D}$ object arrangements. ACM Transactions on Graphics. 31-135.

Gee AP, Escamilla-Ambrosio PJ, Webb M, Mayol-Cuevas W, Calway A. (2010). Augmented crime scenes: virtual

(C) 2018 ADFSL annotation of physical environments for forensic investigation. Proceedings of the 2nd ACM workshop on Multimedia in forensics, security and intelligence. 105-10.

Gibson S, Howard T. (2000). Interactive reconstruction of virtual environments from photographs, with application to scene-ofcrime analysis. ACM Symposium on Virtual Reality Software and Technology. 41-8.

González-Jorge H, Zancajo S, GonzálezAguilera D, Arias P. (2015). Application of Kinect gaming sensor in forensic science. Journal of forensic sciences.60:206-11.

Hołowko E, Januszkiewicz K, Bolewicki P, Sitnik R, Michoński J. (2016). Application of multi-resolution 3D techniques in crime scene documentation with bloodstain pattern analysis. Forensic science international. (267):218-27.

Howard TL, Murta AD, Gibson S. (2000). Virtual environments for scene of crime reconstruction and analysis. Electronic Imaging: International Society for Optics and Photonics. 41-48.

Jancosek M, Pajdla T. (2011). Multi-view reconstruction preserving weakly-supported surfaces. Computer Vision and Pattern Recognition (CVPR), 3121-3128.

eTeks. (2017). Homepage of Sweet home 3D, Retrieved on October 21, 2017 from http://www.sweethome3d.com/.

Leipner A, Baumeister R, Thali MJ, Braun M, Dobler E, Ebert LC. (2016). Multi-camera system for 3D forensic documentation. Forensic science international. 261:123-8.

Maksymowicz K, Tunikowski W, Kościuk J. (2014). Crime event 3D reconstruction based on incomplete or fragmentary 
evidence material-Case report. Forensic science international. 242: e6-e11.

Ma M, Zheng H, Lallie H. (2010). Virtual reality and 3D animation in forensic visualization. Journal of forensic sciences. 55:1227-31.

Qian L, Fan Y, Zhou M, et al. (2016). Manifold Ranking for Sketch-Based 3D Model Retrieval. Transactions on Edutainment XIII. Springer Berlin Heidelberg,149-164.

Sansoni G, Cattaneo C, Trebeschi M, Gibelli D, Poppa P, Porta D, et al. (2011). Sceneof-Crime Analysis by a 3-Dimensional Optical Digitizer: A Useful Perspective for Forensic Science. The American journal of forensic medicine and pathology. 32:280-6.

Tung ND, Barr J, Sheppard DJ, Elliot DA, Tottey LS, Walsh KA. (2015). Spherical Photography and Virtual Tours for Presenting Crime Scenes and Forensic Evidence in New Zealand Courtrooms. Journal of forensic sciences. 60:753-8.

Zobel J, Moffat A. (2006). Inverted files for text search engines. ACM computing surveys (CSUR). 38:6-18. 